\title{
Object-centered attentional biases and object recognition contributions to scene segmentation in left- and right-hemisphere-damaged patients
}

\author{
MARY A. PETERSON and PETER C. GERHARDSTEIN \\ University of Arizona, Tucson, Arizona \\ MARK MENNEMEIER \\ University of Alabama, Birmingham, Alabama \\ and \\ STEVEN Z. RAPCSAK \\ University of Arizona, Theson, Arizona
}

\begin{abstract}
Participants viewed elongated rectangular displays in which two regions shared a central contour. In experimental stimuli, the central contour portrayed a known object on one, high-denotative, side. In control stimuli, no known objects were portrayed on either side of the central contour, but one side of each control stimulus was a scrambled version of one of the high-denotative regions, matching it on all factors known to influence scene segmentation other than object recognition. For each display, participants decided whether the left or the right region was more likely to be an object. Paradoxically, both right-hemisphere- $(\mathrm{RH})$ and left-hemisphere- $(\mathrm{LH})$ damaged individuals were more likely to see objects lying on the contralesional rather than the ipsilesional side of the central contour. This tendency is attributed to an object-centered attentional bias toward the central contour when objects lie on its contralesional side and away from the central contour when objects lie on its ipsilesional side. Object-centered attentional biases were stronger following $\mathrm{RH}$ than $\mathrm{LH}$ damage. Elderly control participants showed a slight bias in the same direction as RH-damaged individuals. More high-denotative regions than scrambled regions were seen as objects, even when object-centered attention was biased away from the central contour carrying the object recognition information. The latter result suggests that the object recognition processes contributing to scene segmentation are preattentive.
\end{abstract}

\section{Attentional Biases}

Spatial attention. Attentional biases toward the side of space ipsilateral to a lesion (ipsilesional space) are often evident following brain damage. When such biases are severe, spatial locations contralateral to the lesion (contralesional space) may be ignored or "neglected." Such "unilateral" neglect is more likely to arise from right-hemisphere $(\mathrm{RH})$ lesions than from left-hemisphere (LH) lesions (DeRenzi,

\footnotetext{
We thank our participants for their willingness to take part in this experiment. Thanks also to Pelagie M. Beeson for giving us access to some of the left-hemisphere-damaged participants; to Cindi May for making the elderly participants available to us; to Leticia Carrasco and Josephine Korchmaros for testing the elderly participants; to Elsie Vezey for testing some of the brain-damaged patients; and to Alyson English and Mandee Eckersley for helping to score data. Portions of this work were conducted while M.A.P. was supported by an SBSRI Research Professorship at the University of Arizona and P.C.G. was supported by a postdoctoral fellowship through the McDonnell-Pew Cognitive Neuroscience Center Program Grant to the University of Arizona. P.C.G. is now at the State University of New York, Binghamton. M.M. is in the Department of Physical Medicine and Rehabilitation at the University of Alabama, and S.Z.R. is in the Department of Neurology at the University of Arizona. Correspondence should be addressed to $M$. A. Peterson, Department of Psychology, University of Arizona, Tucson, AZ 85721 (e-mail: mapeters@u.arizona.edu).
}

1982; Ogden, 1987). Unilateral neglect is frequently identified by a bedside battery in which patients will evidence behaviors such as left-sided omissions on line cancellation tasks, constriction of numbers to the right side in clock drawing, and/or placement of line bisection marks to the right of center. Patients with unilateral neglect have intact visual fields; therefore, neglect appears to be a disorder of attention rather than perception.

Hemispheric specialization for spatial attention. Neglect disorders suggest that the undamaged hemispheres may be differentially specialized for allocating attention to spaces located to the left and right of the viewer's midline. Specifically, unilateral neglect might reflect a contralateral attentional orienting bias of the intact hemisphere. The fact that unilateral neglect occurs more often for $\mathrm{RH}$-damaged patients than for $\mathrm{LH}$-damaged patients has been taken to suggest that the attentional orienting bias of the LH is stronger (Kinsbourne, 1970), or more lateralized (Heilman \& Van Den, 1980), than that of the RH. Consistent with these ideas, contralateral attentional biases can be observed in normal (i.e., non-brain-damaged) controls when the $\mathrm{LH}$ and $\mathrm{RH}$ are selectively activated, at least initially, by stimulus presentations in the right or left visual field, respectively (or in right and left hemispace, re- 
spectively) (e.g., Mennemeier, Vezey, Chatterjee, Rapcsak, \& Heilman, 1997; Milner, Brechmann, \& Pasgliarini, 1992; Reuter-Lorenz, Kinsbourne, \& Moscovitch, 1990). Under some conditions used to test normal observers, the contralateral bias exerted by the LH can be observed to be stronger than that exerted by the $\mathrm{RH}$ (Reuter-Lorenz et al., 1990). This last finding is consistent with the clinical evidence that unilateral neglect occurs more commonly following lesions of the RH rather than the LH.

Object-centered attentional biases. In addition to spatial attention, there exists a separate type of attention that is centered on an object's midline rather than on the viewer's midline. That is, brain-damaged patients may neglect the contralesional side of an object regardless of where that object is located in space (Driver \& Halligan, 1991). For example, Gainotti, Messerli, and Tissot (1972) asked a unilateral neglect patient with RH damage to copy a series of simple, familiar objects arrayed from left to right across a page. The patient attempted to copy all of the objects; hence, contralesional spatial neglect was not observed. However, the patient copied only the right side of each object; hence, contralesional neglect was expressed with respect to each object's midline, rather than with respect to the observer's midline.

More recently, Behrmann and Tipper (1994; Tipper \& Behrmann, 1996) demonstrated that, for RH-damaged patients with unilateral neglect, the handedness assigned to the sides of an object in the original view is maintained after the object is rotated $180^{\circ}$ in the picture plane so that the left and right sides of the object swap places. By using this rotation manipulation, Behrmann and Tipper were able to contrast predictions about the direction of attentional bias made on a spatial attention account and an object-centered attention account. Under these conditions, Behrmann and Tipper were able to observe both spatial and object-centered attentional biases in a single patient. These results were important in demonstrating that attention could operate within an object-centered frame as well as in a spatial frame.

The rotation manipulation can be used to contrast predictions from object-centered and spatial attention accounts only for displays in which the left and right sides are defined intrinsically (i.e., with respect to a typical, or the original, view of the object). (For experiments in which handedness of displays was defined with respect to the typical orientation of the object, see Behrmann \& Moscovitch, 1994, and Farah, Brunn, Wong, Wallace, \& Carpenter, 1990.) Another way to isolate object-centered attention is to use displays in which two adjacent regions share a common vertically oriented articulated contour (see Figures 1A and 1B). For such figure-ground displays, spatial attentional biases and object-centered attentional biases operate in opposite directions when the display remains in a single orientation.

Consider first the predictions that would be made for the perception of displays like Figures $1 \mathrm{~A}$ and $1 \mathrm{~B}$ if spatial attention orienting biases affect response. If left and
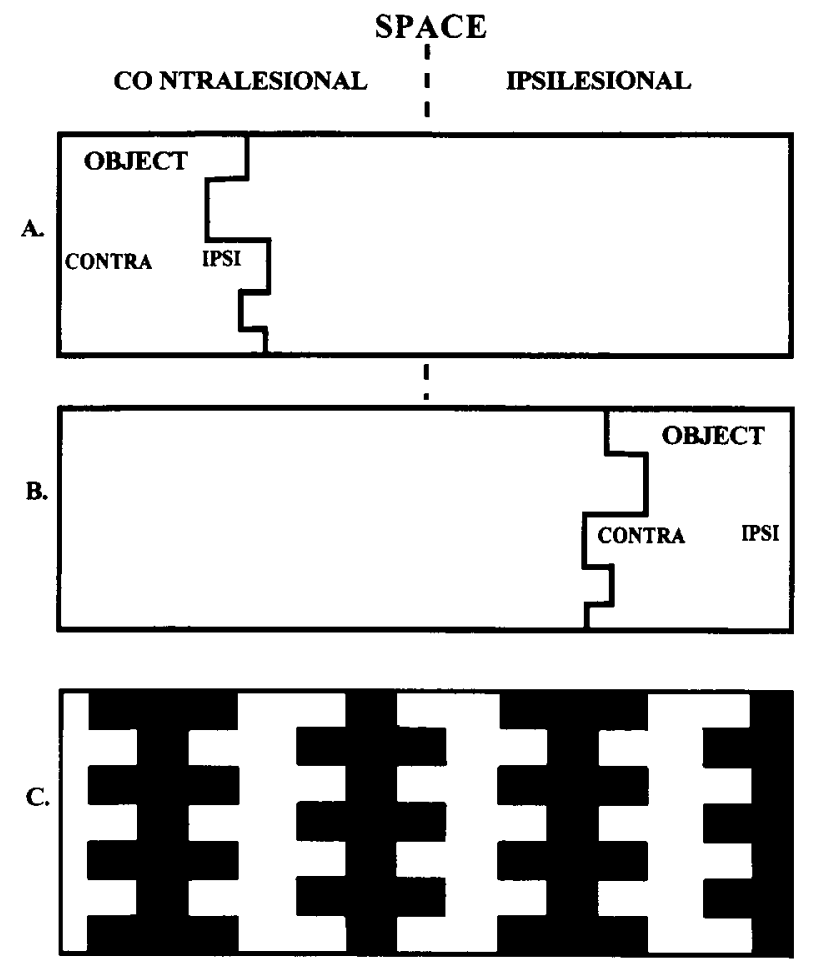

Figure 1. Panels $A$ and $B$ are schematic versions of the stimuli used by Driver, Baylis, and Rafal (1992). For both spaces and objects, the labels contralesional and ipsilesional are appropriate for right-hemisphere-damaged patients. In panel $A$, smallness of relative area favors the interpretation that the left region, located in contralesional space, is the object. The object itself can be divided into contralesional and ipsilesional sides, as indicated. For objects lying on the left, inattention to the contralesional side does not affect perception of the articulated contour, lying on the ipsilesional side. In panel B, smallness of relative area favors the interpretation that the right region, located in ipsilesional space, is the object. For objects lying on the right, inattention to contralesional sides does affect the perception of the articulated contour, which lies on the contralesional side of the figure. Panel $\mathrm{C}$ is a figureground display in which black, symmetric regions alternate with white, asymmetric regions. Symmetric regions are typically seen as objects.

right are defined spatially with respect to the viewer's midline (or with respect to the shared, central, contour ${ }^{1}$ ), RH-damaged patients with unilateral spatial neglect would be expected to neglect the region lying on the left of the shared contour. Consequently, they would be expected to perform better when tasks direct their attention to right regions rather than to left regions. This pattern of behavior would be expected to follow from the rightward spatial attention orienting bias of the intact LH.

Consider next the predictions that would be made for the perception of figure-ground displays like Figures $1 \mathrm{~A}$ and $1 \mathrm{~B}$ if object-centered attentional orienting biases affect response. In order to do so, we must first consider how objects are perceived in displays in which two adjacent regions share a contour (or border), as they do in Figures $1 \mathrm{~A}$ and $\mathrm{lB}$. Contours shared by two regions are 
often assigned to one region only as a result of scene segmentation processes. The region to which the contour is assigned becomes the figure and appears to have a definite shape; the other region is shapeless. Figures are the objects in the display; the adjacent, shapeless, regions are backgrounds (at least locally ${ }^{2}$ ). Eye fixations and attention are usually allocated to figures/objects rather than to their backgrounds (Hochberg, 1971; Peterson \& Gibson, 1994b; Rubin, 1915/1958). The Gestalt psychologists identified a number of cues that affect the assignment of object status. Smallness of relative area is one of them (for listing of configural cues, see Harrower, 1936; Hochberg, 1971). Consequently, in Figure 1 A, the left region is typically seen as the object because it is smaller in area than the right region. By virtue of the same configural cue, the right region should be seen as the object in Figure 1B.

Now consider predictions from an object-centered attention account about how brain damage would affect the orientation of attention within the objects perceived in Figures $1 \mathrm{~A}$ and $1 \mathrm{~B}$. For RH-damaged patients, the right (ipsilesional) side of the object is the side to which attention is preferentially oriented as a consequence of brain damage (presumably because of the contralateral objectcentered orienting bias of the intact LH). Therefore, on an object-centered attentional account, RH-damaged patients should be able to see the shape of objects lying on the left side of the central contours (as in Figure 1A) quite well. That is because the contour endowing the left object with a definite shape lies on its right side. Conversely, objects lying on the right of the central contour (as in Figure 1B) have a definite shape because of the contour lying on their left side. For RH-damaged patients, the left (contralesional) side of the object is the side that is often neglected following RH damage (again, presumably because of the contralateral object-centered orienting bias of the intact $\mathrm{LH}$ ). Thus, on an object-centered attentional account, RH-damaged patients would be expected to perform poorly on any task requiring them to see the specific shape of objects lying on the right side of the central contour.

Thus, for figure-ground displays like those in Figures $1 \mathrm{~A}$ and $1 \mathrm{~B}$, opposite predictions about direction of attentional bias follow from a spatial attention account and an object-centered attention account when orientation remains unchanged. Notice that the assignment of left and right directions to the individual objects perceived in Figures $1 \mathrm{~A}$ and $\mathrm{B}$ derives from the viewer's left and right sides. Nevertheless, these directions are object centered rather than spatial because they are assigned relative to the object's midline (or axis; Driver, Baylis, Goodrich, \& Rafal, 1994) rather than relative to the viewer's midline.

Consistent with the object-centered attention predictions, RH-damaged patients with unilateral neglect who attempted to copy or to match the central contour performed better on displays like Figure 1A than on displays like Figure 1B (Driver, Baylis, \& Rafal, 1992; Marshall \& Halligan, 1994; Mattingly, Price, \& Driver, 1996).
Hemispheric specialization for object-centered attention. Reports of object-centered attentional biases have been infrequent, and most of the patients reported to show object-centered attentional biases have been unilateral neglect patients with RH damage (see, e.g., Behrmann \& Moscovitch, 1994; Behrmann \& Tipper, 1994; Driver et al., 1994; Driver et al., 1992; Driver \& Halligan, 1991; Farah et al., 1990; Marshall \& Halligan, 1994; Mattingly et al., 1996; Tipper \& Behrmann, 1996). This practice might lead prematurely to the belief that object-centered neglect is more likely to be found in RHdamaged than LH-damaged patients, and that in RHdamaged patients, object-centered neglect is likely to cooccur with spatial neglect. (For a similar argument, see Mennemeier et al., 1997). Caramazza and Hills (1990) and Humphreys and Riddoch (1994) did observe objectcentered attentional biases in unilateral neglect patients with LH damage. On the basis of their research, Humphreys and Riddoch hypothesized that object-centered attentional biases might be found more often following LH damage than RH damage. ${ }^{3}$

Obviously, additional evidence regarding hemispheric specialization for object-centered attention is required. As a step in that direction, the present experiment uses figure-ground displays to investigate object-centered attentional biases in both RH-damaged and $\mathrm{LH}$-damaged individuals. The first question addressed in this article is whether object-centered attentional biases are more likely to be observed, or are stronger, following $\mathrm{RH}$ damage than LH damage, as spatial attentional biases are; or whether the hemispheric specialization for object-centered attention is reversed, as Humphreys and Riddoch (1994) proposed. In addition, we examined $\mathrm{RH}$-damaged individuals both with and without unilateral spatial neglect in order to investigate whether object-centered attentional biases and clinically observable unilateral spatial neglect necessarily co-occur.

Another question addressed in the present article is whether or not hemispheric specialization for objectcentered attention can be observed when normal observers view figure-ground displays. Reuter-Lorenz, Drain, \& Hardy-Morais (1996) used a gap detection task to investigate whether or not object-centered attentional biases were lateralized in normal observers. ${ }^{4}$ They positioned the left and right sides of objects shown on different trials on identical retinotopic locations in either the left or the right visual field (LVF or RVF, respectively). With target eccentricity held constant in this way, they found that observers were more likely to detect gaps located on the right side of an object when the object was viewed in the RVF (LH) than in the LVF (RH). Conversely, observers were more likely to detect gaps located on the left side of an object when the object was viewed in the LVF (RH) than in the RVF (LH). These results suggest that the two hemispheres may be specialized for attending to the contralateral sides of objects (as well as to contralateral spatial locations; see Reuter-Lorenz, Kinsbourne, \& Mosco- 
vitch, 1990). Unlike the previous experiments investigating spatial attention conducted by Reuter-Lorenz et al. (1990), the experiments investigating object-centered attentional biases revealed no evidence that biases were stronger in one hemisphere than the other.

In the experiment reported here, we used figure-ground displays and an object decision task to assess objectcentered attentional biases in normal elderly control observers who were matched in age to the brain-damaged participants. Long display durations were used, unlike the brief display durations used by Reuter-Lorenz et al. (1996) in their tests of normal young observers. It is unusual to find evidence for hemispheric specialization in normal young observers using long exposure conditions. Consequently, the conditions used in the present experiment may not be a sensitive test of hemispheric specialization in normal observers. However, recent evidence indicates that, for some visual tasks, elderly observers show a larger age-related deficit when the RH is activated than they do when the LH is activated (Gerhardstein, Peterson, \& Rapcsak, 1998; Polster \& Rapcsak, 1994; Stark \& Coslett, 1993). If figure-ground tasks tap into brain structures that decline with age, then object-centered attentional biases similar to those revealed by $\mathrm{RH}$-damaged individuals may be evident in our elderly control observers, even under long exposure conditions.

\section{The Task}

The figure-ground displays and the object decision task used in this paper were introduced as a test for braindamaged patients by Peterson, Rapcsak, and Gerhardstein (1994; see also Peterson, de Gelder, Rapcsak, Gerhardstein, \& Bachoud-Lévi, 1998). The object decision task was adapted from tasks used with young normal observers in research investigating whether or not processes entailing access to memories of objects (i.e., a subset of object recognition processes) affect which of two adjacent regions will attain object status during scene segmentation processes (Gibson \& Peterson, 1994; Peterson, 1994b; Peterson, Harvey, \& Weidenbacher, 1991). Recently, Mattingly et al. (1996) used some of these stimuli to demonstrate object-centered attentional biases in a unilateral neglect patient with RH damage.

The stimulus displays comprised two adjacent regions sharing a contour. Half the stimuli were experimental stimuli. In the experimental stimuli, one region depicted a mono-oriented familiar object when it was seen as the figure/object, it was identified correctly by at least $65 \%$ of pilot observers. Therefore, it was assumed that this "high-denotative" region provided a good match to a representation of a known object in memory. High-denotative regions occurred equally often on the left and right sides of the shared central contour (see Figures 2A and 2B). The region adjacent to the high-denotative region was "low denotative" in that it did not depict a known object when it was seen as the object. (Fewer than $22 \%$ of control observers agreed on a single interpretation for any of the low-denotative regions when they were seen as ob- jects.) Control stimuli constituted the other half of the stimuli. In control stimuli, both adjacent regions were low in denotivity. However, one region was matched to the high-denotative region of the experimental stimuli in that it was constructed by spatially rearranging (or "scrambling") the parts of a high-denotative region (see Figures $2 \mathrm{C}$ and $2 \mathrm{D}$ ). The scrambled regions matched the highdenotative regions in part structure ${ }^{5}$ but, when scrambled regions appeared to be objects, they were not recognizable as known objects any more often than the other lowdenotative regions were. Therefore, we assumed that scrambled regions did not provide a good match to memory representations coding the structure of the object. Like high-denotative regions, scrambled regions occurred equally often on the left and right sides of the shared central contour.

In the present experiment, brain-damaged and control observers were shown stimuli like those in Figure 2 one at a time and asked to identify any known object they saw. When they did not see a known object, they were asked to decide which region was more likely to be an object (object decision). High-denotative and scrambled regions were equated on variables known to be relevant to figure-ground assignment, but differed in their goodness of fit to object representations. Therefore, any tendency to see high-denotative regions as objects more often than scrambled regions was taken to indicate that object recognition processes contribute to scene segmentation.

In previous experiments, conducted with college students, high-denotative regions were more likely than scrambled regions to be seen as objects (Gibson \& Peterson, 1994; Peterson et al., 1991). These effects were obtained under both brief- and long-exposure conditions, and when observers reported about the first-perceived figure-ground assignment or about reversals of perceived figure-ground status. On the basis of these previous experiments, ${ }^{6}$ proposals were made about how these object recognition effects on scene segmentation were mediated. Those proposals will be summarized in the next section. The next section will also include a discussion of how data obtained from brain-damaged patients can provide important evidence about the nature of these object recognition contributions to scene segmentation.

\section{Object Recognition Contributions to Scene Segmentation}

In order to account for the evidence that some object recognition processes contribute to scene segmentation, Peterson and her colleagues (Peterson, 1994a, 1994b, in press; Peterson \& Gibson, 1993, 1994a, 1994b) proposed that object recognition processes begin to operate upon edges shortly after they are detected in the visual array. On this view, object representations are accessed very early in visual processing. The proposal is that these object recognition processes are conducted in parallel with processes assessing other figure-ground cues (e.g., symmetry, smallness of relative area, and convexity). ${ }^{7}$ Provided that the outputs from these critical object recognition pro- 


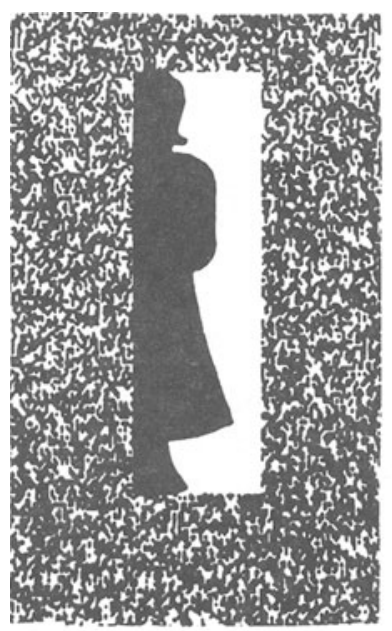

A

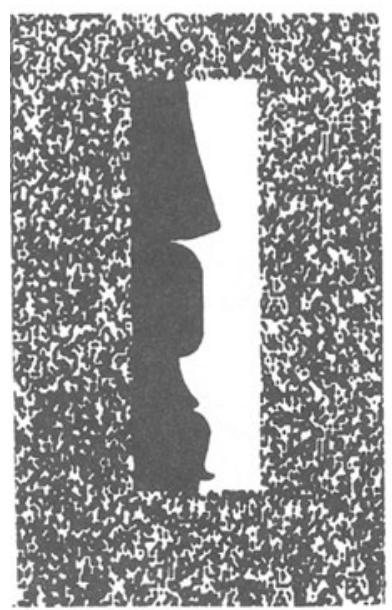

C

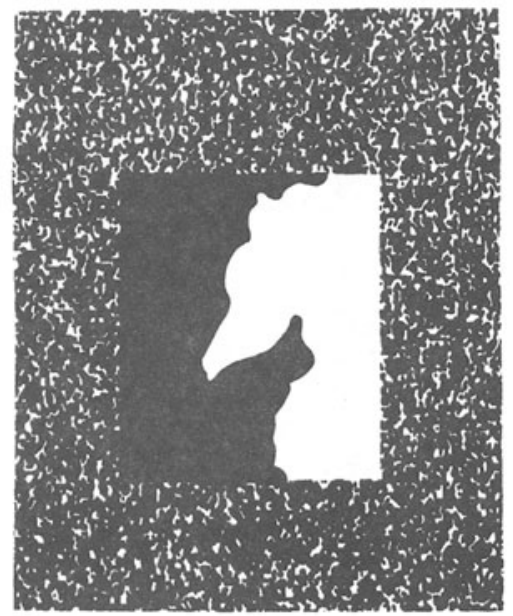

B

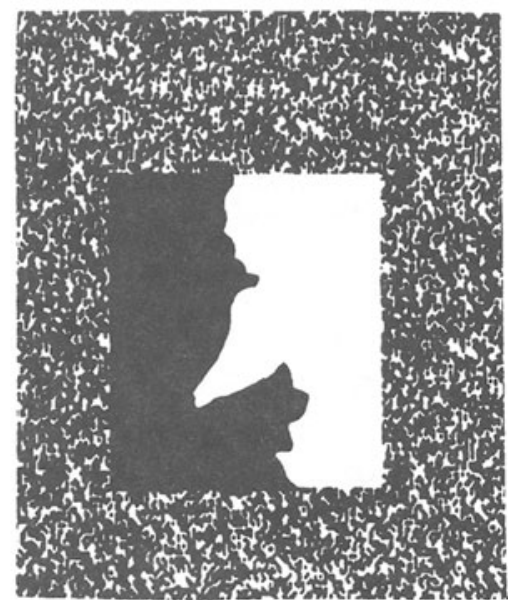

D

Figure 2. Sample experimental (panels $A$ and $B$ ) and control figure-ground displays. The high-denotative regions portray a standing woman (panel $A$, left, black region) and a sea horse (panel $B$, right, white region). Panels $C$ and $D$ are the control stimuli containing scrambled versions of the high-denotative regions in panels $A$ and $B$, respectively.

cesses are available quickly, they can combine with the outputs of the processes assessing other figure-ground cues to determine which region will be seen as the object.

The proposals made by Peterson et al. (Peterson, 1994a, 1994b, in press; Peterson \& Gibson, 1993, 1994a, 1994b) are controversial because they challenge a long-standing assumption that object recognition processes operate only after figures/objects have been separated from shapeless grounds (fully or partially) (see, e.g., Köhler, 1929/ 1947; Neisser, 1967; Palmer \& Rock, 1994; Vecera \& O'Reilly, 1998; Wallach, 1949). Hence, alternative interpretations of our results have been offered. One alternative interpretation is that in our previous experiments, the high-denotative regions were seen as objects more often than the scrambled regions because the high-denotative regions were more likely to be recognized consciously. It is true that conscious recognition occurs for figures/objects and not for grounds (Hoffman \& Richards, 1985; Rubin, 1915/1958). Consequently, in the previous experiments, it is likely that conscious recognition often accompanied observers' reports that the high-denotative regions appeared to be figures/objects. If conscious object recognition necessarily accompanies reports that the highdenotative regions appear to be figures/objects, it might be impossible to rule out this alternative interpretation.

Recently, Peterson et al. (1998) obtained results that argue against the alternative interpretation just described. They found that a visual agnosic patient whose conscious 
object recognition was severely impaired nevertheless demonstrated robust contributions from object recognition processes to scene segmentation processes. The argument against the alternative account would be stronger if other evidence suggested that the object recognition processes critical for scene segmentation operate preattentively, as other scene segmentation processes do. This is where tests of brain-damaged individuals who show object-centered attentional biases are relevant.

Preattentive processing. The idea that tests of braindamaged patients might be useful for investigating whether the object recognition processes relevant to scene segmentation are preattentive derives from previous work by Driver and his colleagues (Driver et al., 1992; Mattingly et al., 1996) and by Marshall and Halligan (1994). Driver et al. (1992) demonstrated that the same RH-damaged patient who neglected the left side of objects in displays like Figure 1B nevertheless saw the symmetric regions in displays like Figure 1C as objects more often than expected on the basis of chance. Symmetry is a scene segmentation cue that requires a comparison across the left and right sides of a region. Like all traditional scene segmentation cues, symmetry is thought to be computed preattentively, before object status is determined (Neisser, 1967). Thus, the results reported by Driver et al. (1992) indicated that both sides of the regions in their displays were treated normally by preattentive processes, even though neglect was applied to one side of the objects that were perceived as a result of those preattentive processes (see also Marshall \& Halligan, 1994).

Following from these experiments conducted by Driver and his colleagues (Driver et al., 1992; Mattingly et al., 1996), we reasoned that we could investigate whether the object recognition processes affecting scene segmentation were preattentive by testing brain-damaged individuals who demonstrated object-centered attentional biases. In the displays shown in Figures $2 \mathrm{~A}$ and $2 \mathrm{~B}$, the information critical for object recognition processes is carried by the central contour, which lies on the right side of objects seen on the left of the display and on the left side of objects seen on the right of the display. For brain-damaged participants, the central contour is the contralesional contour of ipsilesional objects. When object-centered attentional biases occur as a consequence of brain damage, attention is biased away from the contralesional contours of objects. Therefore, the performance with ipsilesional regions constitutes the critical test of whether or not the object recognition processes contributing to scene segmentation are preattentive. If the high-denotative ipsilesional regions are seen as objects more often than scrambled ipsilesional regions, even though there exists an attentional bias toward seeing contralesional regions as objects, that will suggest that the object recognition processes producing the preference for high-denotative regions are preattentive. Alternatively, if high-denotative and scrambled ipsilesional regions are seen as objects approximately equally often, the interpretation that object recognition processes are preattentive is less likely.

\section{Summary}

In summary, the object decision task described above will allow us to (1) investigate the strength of objectcentered attentional biases in both $\mathrm{RH}$ - and LH-damaged participants and (2) investigate whether those object recognition processes that affect scene segmentation operate preattentively, as other processes relevant to scene segmentation do. Object-centered attentional biases will be evident if brain-damaged participants show a bias to see contralesional regions over ipsilesional regions as objects. The critical evidence regarding the preattentive nature of the object recognition processes contributing to scene segmentation will be found in responses about ipsilesional regions. If ipsilesional high-denotative regions are seen as objects more often than ipsilesional scrambled regions, that will suggest that the critical object recognition processes are preattentive.

\section{METHOD}

\section{Participants}

The participants were 8 RH-damaged males (mean age $=67$ years), 8 LH-damaged individuals ( 7 males, 1 female; mean age $=$ 55 years), and 12 male control observers (mean age $=70$ years). Lesion location and ages are listed for each brain-damaged participant individually in Table 1. Five of the RH-damaged participants were judged to have neglect clinically, indicated by the asterisks next to those patients' initials in the table. Four of these patients participated in a line bisection study (Mennemeier et al., 1997). None of the LH-damaged participants showed clinical signs of neglect; none of the LH-damaged participants was formally tested for neglect. The control participants volunteered to participate in this study after taking part in a memory experiment at the University of Arizona. None of the control participants reported head injury.

\section{Stimuli}

Six different sets of figure-ground displays were used. Four of the sets comprised 54 stimuli, arranged into three subsets of 18 stimuli, including 10 experimental and 8 control stimuli. The two other sets comprised 48 stimuli, arranged into two subsets of 24

Table 1

\begin{tabular}{|c|c|c|}
\hline \multicolumn{3}{|c|}{ Patient Information } \\
\hline Patient & Age & Lesion Location \\
\hline \multicolumn{3}{|r|}{ Left Hemisphere } \\
\hline M.L. & 45 & Fronto-temporo-parietal \\
\hline E.D. & 40 & Temporo-parietal \\
\hline O.P. & 77 & Parieto-occipital \\
\hline S.V. & 44 & Temporal lobe, insula, and frontal lobe \\
\hline S.W. & 42 & Temporal lobe \\
\hline J.H. & 68 & Temporo-occipital \\
\hline H.L. & 56 & Occipito-temporal \\
\hline G.K. & 64 & Fronto-temporo-parieto-occipital \\
\hline \multicolumn{3}{|r|}{ Right Hemisphere } \\
\hline C.C. & 66 & Temporo-occipital \\
\hline T.H. & 67 & Temporo-parieto-occipital \\
\hline W.O. & 59 & Temporal \\
\hline L.U.* & 68 & Parieto-frontal \\
\hline R.S.* & 58 & Temporo-parietal \\
\hline B.H.* & 71 & Fronto-temporo-parietal \\
\hline W.A.* & 80 & Frontal, large intracerebral \\
\hline W.J.* & 69 & Fronto-temporo-parietal \\
\hline
\end{tabular}

*Patients with clinically assessed unilateral neglect. 
stimuli, including 12 experimental and 12 control stimuli. ${ }^{8} \mathrm{~A}$ given experimental stimulus and its corresponding scrambled control stimulus were not included in the same subset.

Scrambled regions were created from high-denotative regions by breaking the central contour of the high-denotative regions into parts delimited by two successive concave cusps along the contour. These parts were rearranged spatially by hand (maintaining their polarity) until the resulting scrambled region was low in denotivity (i.e., failed to elicit greater than $22 \%$ between-subjects agreement on what object it portrayed in a pilot experiment). Scrambled regions of some of the high-denotative regions in each set failed to meet this criterion; scrambled regions of other high-denotative regions not included among the experimental stimuli were substituted in their stead.

Black/white (B/W) color and right/left $(\mathrm{R} / \mathrm{L})$ location of the highdenotative and the scrambled regions were balanced within and across sets. The stimuli ranged in width from 3.5 to $8.9 \mathrm{~cm}$ and in height from 8.0 to $13.6 \mathrm{~cm}$. Each B/W stimulus was drawn on a white sheet of $21.25 \times 27.5 \mathrm{~cm}$ paper and surrounded by a frame of $B / W$ random dots, approximately $16 \times 19.5 \mathrm{~cm}$, which left a second white frame surrounding the $\mathrm{B} / \mathrm{W}$ frame. Each sheet of paper was laid on top of a second sheet of $23 \times 30.5 \mathrm{~cm}$ black paper that provided a third, black, frame. These three frames were employed to eliminate any bias toward seeing black regions as figure that might have been present had the pictures been presented on a plain white background.

A familiarization set comprised eight stimuli. Six of the familiarization stimuli depicted whole known objects in either white $(n=$ 3 ) or black $(n=3)$ silhouette on a contrasting ground surrounded by the three frames described above. The whole objects in the familiarization set were closed and smaller in area than their surrounds so that the configural cues of closure and relative area would operate to specify that they were seen as figures rather than grounds (Harrower, 1936; Hochberg, 1971; Rubin, 1915/1958). The objects depicted in the familiarization set were a cat, a windmill, a map of the state of Texas, a hatchet, and an airplane. Two additional familiarization stimuli, shown last, were half-versions of the whole objects (the cat and the windmill) in the same style as those shown in Figures $2 \mathrm{~A}$ and $2 \mathrm{~B}$.

\section{Procedure}

Participants were tested one at a time at the Veterans' Administration Medical Center in Tucson, the University of Arizona Health Sciences Center, or the University of Arizona by one of seven experimenters, including the four authors. All stimuli were placed on a tabletop in front of the participant (viewing distance was $30-50 \mathrm{~cm}$ ). The experimenter began by asking the participants to identify a set of 18 silhouette objects and/or the 6 whole objects in the familiarization set. These silhouettes were created by filling in the contours of line drawings gathered from a number of different sources (e.g., children's coloring books, the Snodgrass and Vanderwart, 1980, set). All participants showed normal recognition abilities in this pretest.

The experimenter then showed the participants the two half-stimuli in the familiarization set and pointed out the regions portraying half of two of the known objects they had just identified. Next, the experimenter told the participants that they would be viewing a set of test stimuli that, like the last two stimuli in the familiarization set, would comprise a black and a white region on either side of a central contour within a rectangular frame. The experimenter stressed that many of the test stimuli would not portray a known object; they were novel objects that had been drawn by the experimenters. Participants were asked to identify any known objects they saw and to indicate whether the object they identified was black or white. They were told that when they could not recognize anything familiar, they were to decide which region looked more like it could be an object (object decision). Object decision reports could be made either by pointing or by responding verbally ("left"//right" or "black"/"white"). Observers were encouraged to respond in terms of color $(B / W)$.
When we began this set of studies, we were interested in spatial neglect. Accordingly, we showed 8 of the brain-damaged participants ( $2 \mathrm{LH}$-damaged and $6 \mathrm{RH}$-damaged participants) the three subsets of the 54 stimulus set in one of three spatial locations with respect to their bodies: either centered on their midline or shifted so that the inner edge of the paper was located approximately $30 \mathrm{~cm}$ to the left or the right of the midline. Set, order, and location of the subsets were balanced across these participants. No differences in performance across the three different spatial locations were found in the patients included in this study, ${ }^{9}$ although object-centered attentional biases were clearly present. ${ }^{10}$ The remaining participants (6 $\mathrm{LH}$-damaged and $2 \mathrm{RH}$-damaged, as well as all control participants) viewed the stimuli in the center location only.

\section{Data Analysis}

There were two dependent variables: object decisions and identification accuracy. Each is described below.

Object decisions. The proportions of object decisions for highdenotative and scrambled regions were calculated separately for regions lying on the left and the right of the central contour. Because object identification cannot occur unless a region appears to have a definite shape (Hoffman \& Richards, 1985; Peterson et al., 1991; Rubin, 1915/1958), any attempt to identify a region was considered an object decision favoring that region. These proportions were entered into an analysis of variance (ANOVA) with one betweensubjects factor (group: LH-damaged, RH-damaged, and control) and two within-subjects factors (region type: high denotative vs. scrambled; and side of contour: L vs. R).

In addition, for each observer, we calculated the proportion of trials on which left regions were chosen as objects, averaging over high-denotative regions, scrambled regions, and low-denotative complements to high-denotative and scrambled regions lying on the right side of the contour. Three planned comparisons were conducted on these proportions. First, we tested whether control participants reported seeing left regions as objects more often than expected on the basis of chance. Next, we separately compared the object decisions made by LH-damaged and RH-damaged participants with those of control participants to see if the brain-damaged participants showed any evidence of object-centered or spatial attentional biases.

Identification accuracy. Identification accuracy was assessed as the proportion of trials on which participants accurately identified the objects depicted by the high-denotative regions. A liberal criterion was used for scoring identification responses. For example, responses of "sea horse," "horse," and "dragon" were scored as correct for the high-denotative region portraying a sea horse, shown in Figure 2B. Also considered correct were descriptions of object function or gestures (e.g., 1 LH-damaged participant held his hand and arm as if he were holding an umbrella when shown a highdenotative region depicting an umbrella).

Simple identification responses are confounded with object decisions in that conscious object identification occurs only for regions seen as figures/objects (Hoffman \& Richards, 1985; Peterson et al., 1998; Rubin, 1915/1958). Therefore, in order to assess identification accuracy separately from object decisions, we conditionalized identification accuracy on object decisions. An ANOVA was conducted on the conditional identification scores with one betweensubjects factor (group: LH-damaged, RH-damaged, and control) and one within-subjects factors (side of contour: $L$ vs. R).

\section{RESULTS}

\section{Object Decisions}

Planned comparisons on the preference for left regions. Averaged over all region types (high denotative, low denotative, and scrambled), the proportion of object decisions made for left regions by the control participants 
(.53) was significantly larger than expected on the basis of chance $[t(11)=2.078, p<.03]$. RH-damaged participants were even more likely (.69) than control participants to see left (contralesional) regions as objects $[t(18)=$ $4.10, p<.001]$. LH-damaged patients, on the other hand, were less likely (.42) than control participants to see left (ipsilesional) regions as objects $[t(18)=9.41, p<.001]$, preferring instead to see right (contralesional) regions as objects. Thus, in each of the brain-damaged groups, there was a bias to see contralesional regions, rather than ipsilesional regions, as objects.

These preferences are exactly what would be expected on an object-centered attention account. The contours endowing objects lying on the left of the central contour with shape are located on the object's right side. On an object-centered attention account, attention would be biased toward these contours when the RH is damaged, and LH contralateral object-centered attentional biases dominate; attention would be biased away from these contours, toward contours on the object's left, when the LH is damaged, and RH contralateral object-centered attentional biases dominate. That is exactly the pattern of performance we obtained in both RH-damaged and LH-damaged participants. Thus, with respect to the first question addressed in this paper, evidence for object-centered attentional bi- ases was obtained in both RH-damaged and LH-damaged participants.

The pattern of performance shown by most of the participants in each group was consistent with the group means. Seven of the 8 participants in the RH-damaged group and 6 of the 8 participants in the LH-damaged group saw a larger proportion of contralesional than ipsilesional regions as objects. Thus, the prevalence of object-centered attentional biases was similar (although not identical) in the two brain-damaged groups.

The control participants evidenced a slight, but significant, preference for seeing left regions as objects over right regions. We return to this point in the Discussion section. For now, we point out that the direction of preference shown by the elderly control participants was the same as that shown by the RH-damaged patients.

High-denotative and scrambled regions. The mean proportions of object decisions made by the three groups of participants for high-denotative and scrambled regions lying on the left and right sides of the central contour are shown in Figure 3. The ANOVA showed a main effect of region type: The proportion of high-denotative regions seen as objects $(.84)$ was larger than the proportion of scrambled regions $(.68)[F(1,2)=109.27, p<$ $.001]$. Main effects of group and contour side were also

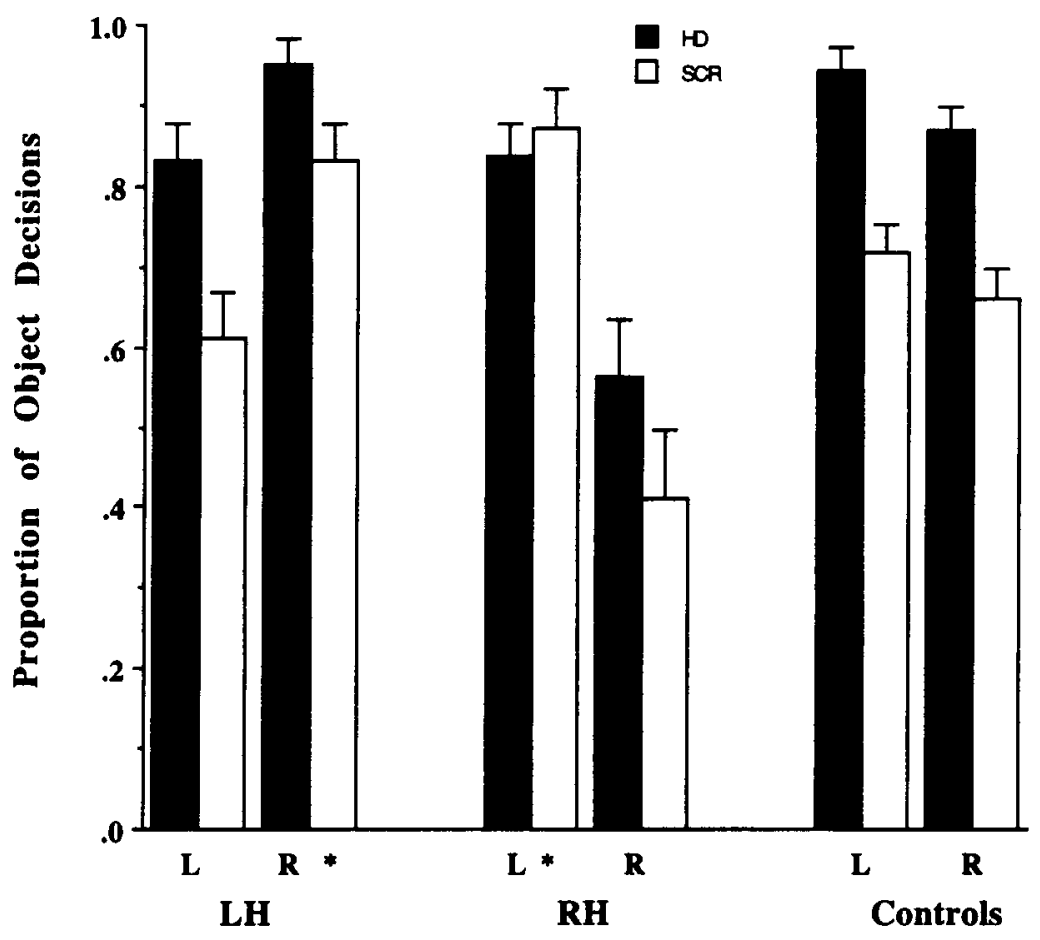

Figure 3. Object decisions made by left-hemisphere (LH)-damaged, righthemisphere (RH)-damaged, and control participants for regions lying on the left (L) and the right $(R)$ of the central contour. For each of the brain-damaged groups, asterisks indicate the contralesional region. Black bars represent high-denotative (HD) regions of experimental stimuli; white bars represent scrambled (SCR) regions of control stimuli. 
Table 2

Proportion of Object Decisions for High-Denotative and Scrambled Regions Side of Contour

\begin{tabular}{|c|c|c|c|c|c|c|}
\hline \multirow[b]{3}{*}{ Group } & \multicolumn{6}{|c|}{ Side of Contour } \\
\hline & \multicolumn{3}{|c|}{ Left } & \multicolumn{3}{|c|}{ Right } \\
\hline & HD & SCR & $M$ & HD & SCR & $M$ \\
\hline
\end{tabular}

Left-Hemisphere Patients

\begin{tabular}{lccccccc} 
& \multicolumn{3}{c}{ Ipsilesional Side } & & \multicolumn{3}{c}{ Contralesional Side } \\
\cline { 2 - 3 } \cline { 6 - 7 } M.L. & .87 & .50 & .76 & & 1.0 & 1.0 & 1.0 \\
E.D. & .83 & .58 & .71 & & 1.0 & .92 & .96 \\
O.P. & .82 & .62 & .72 & & .93 & .90 & .92 \\
S.V. & .92 & .73 & .82 & & .91 & .92 & .91 \\
S.W. & 1.0 & .58 & .79 & & .83 & .75 & .79 \\
J.H. & .56 & .30 & .46 & & .79 & .71 & .75 \\
H.L. & .75 & .85 & .79 & & 1.0 & .91 & .96 \\
G.K. & .93 & .72 & .86 & & .78 & .60 & .74 \\
$M$ & .83 & .61 & .72 & & .90 & .84 & .87 \\
$S E$ & .05 & .06 & .05 & & .03 & .05 & .03
\end{tabular}

Right-Hemisphere Patients

\begin{tabular}{lccccccc} 
& \multicolumn{3}{c}{ Contralesional Side } & & \multicolumn{3}{c}{ Ipsilesional Side } \\
\cline { 2 - 4 } \cline { 6 - 7 } C.C. & .93 & 1.0 & .96 & & .27 & .08 & .18 \\
T.H. & .91 & .91 & .91 & & .62 & .25 & .44 \\
W.O. & .80 & 1.0 & .89 & & .73 & .58 & .67 \\
L.U.* & .93 & .86 & .91 & & .73 & .60 & .70 \\
R.S.* & .92 & .92 & .92 & & .25 & .17 & .21 \\
B.H.* & .87 & .86 & .86 & & .50 & .40 & .47 \\
W.A.* & .67 & .86 & .73 & & .73 & .40 & .65 \\
W.J.* & .67 & .57 & .64 & & .67 & .80 & .70 \\
$M$ & .84 & .87 & .85 & & .56 & .41 & .49 \\
$S E$ & .04 & .05 & .03 & & .07 & .09 & .06 \\
Control Participants & & & & & \\
$M$ & .94 & .72 & .83 & & .87 & .66 & .76 \\
$S E$ & .03 & .04 & .03 & .03 & .04 & .03 \\
\hline
\end{tabular}

Note-HD, high-denotative region of experimental stimuli; SCR, corresponding scrambled region of control stimuli. *Patients who evidenced unilateral neglect clinically.

obtained $[F(2,25)=7.96, p<.003 ; F(1,2)=6.483, p<$ .02 , respectively]. These main effects were moderated by significant two-way interactions between contour side and group and between region type and group $[F \mathrm{~s}(2,15)=$ 14.605 and 12.509 , respectively, $p s<.001]$ and by a threeway interaction among group, region-type, and contour side $[F(2,15)=4.179, p<.03]$. Consequently, post hoc tests were conducted to identify the differences underlying these interactions.

Given the two-way interaction between contour side and group, we conducted a follow-up ANOVA to test whether the proportions of object decisions favoring contralesional regions were different for $\mathrm{RH}$-damaged and LH-damaged groups. This test is relevant to the question of whether object-centered attentional biases are stronger following damage to one of the two hemispheres. Scheffé post hoc tests indicated that the proportions of object decisions made for contralesional high-denotative and scrambled regions were approximately the same in the RH-damaged and LH-damaged groups $(F<1)$ (Figure 3). Table 2 shows that the behavior of individuals in the two groups was consistent with the group means. Seven of the $8 \mathrm{RH}$-damaged patients and 6 of the $8 \mathrm{LH}$-damaged patients made more object decisions for contralesional high-denotative and scrambled than ipsilesional regions. Six of the 7 RH-damaged participants and 5 of the $6 \mathrm{LH}-$ damaged participants who showed this effect chose both the high-denotative and the scrambled contralesional regions as objects on $75 \%$ or more of the possible trials. By this measure-the likelihood of seeing contralesional high-denotative and scrambled regions as objects-objectcentered attentional biases appear to be approximately equivalent in the two hemispheres.

Scheffé post hoc tests showed that the proportion of object decisions favoring ipsilesional high-denotative and scrambled regions was larger in LH-damaged participants than in RH-damaged participants $[F(1,30)=10.02, p<$ $.004]$ (Figure 3). Thus, by this measure - the likelihood of seeing ipsilesional high-denotative and scrambled regions as objects-it seems that object-centered attentional biases may be stronger in RH-damaged than in LH-damaged participants. Instead of choosing ipsilesional high-denotative or scrambled regions as objects, RH-damaged participants were more likely to choose the complementary (contralesional) low-denotative regions as objects. The incidence of the neglect disorder among the RH-damaged participants does not seem to account for their lower relative likelihood of seeing ipsilesional high-denotative and scrambled regions as objects (Table 2). Although the number of participants in the neglect and non-neglect subgroups of RH-damaged participants was too small to permit a statistical test, the individual cases show that neglect is not a necessary condition for this pattern of behavior.

Thus, one measure - the proportion of object decisions favoring contralesional high-denotative and scrambled regions - revealed the object-centered attentional biases to be equally strong in RH-damaged and LH-damaged participants, whereas another measure-the proportion of object decisions favoring ipsilesional high-denotative and scrambled regions - revealed the object-centered attentional biases to be stronger in the $\mathrm{RH}$-damaged participants than in the LH-damaged participants.

Object recognition contributions to figure-ground segmentation. Post hoc examination of the three-way interaction among group, region type, and contour side was conducted by calculating simple effects to compare the proportions of object decisions made by each participant group for high-denotative regions and scrambled regions on each side of the central contour. Object recognition contributions to object status were clearly evident in the control participants. For control participants, the proportion of object decisions was larger for highdenotative regions than scrambled regions on both left and right sides of the contour $[F \mathrm{~s}(2,25)=22.50$ and 18.67 , respectively]. The brain-damaged groups both showed evidence of object recognition contributions to object status for ipsilesional regions, but not for contralesional 
regions, where object decisions favoring both high-denotative regions and for scrambled regions were close to ceiling. For LH-damaged participants, the proportion of object decisions was larger for high-denotative regions than for scrambled regions on the left side of the contour $[F(2,25)=14.50]$, but not the right side of the contour $(F=1.28)$. For RH-damaged participants, the proportion of object decisions was larger for high-denotative regions than for scrambled regions on the right side of the contour $[F(2,25)=14.50]$ but not on the left side $(F<1)$.

All of the control participants saw a larger proportion of high-denotative than scrambled regions as objects on the right side of the contour, and 11 of the 12 control participants showed the same pattern for left regions. The failure of 1 control participant to show an advantage for high-denotative over scrambled regions on the left side was probably due to a ceiling effect: This control participant chose both left regions as objects more than $75 \%$ of the time. The results obtained from individual braindamaged participants reflected those seen in the group means. Seven of the participants in each brain-damaged group saw ipsilesional high-denotative regions as objects more often than ipsilesional scrambled regions. Thus, the majority of the participants in each brain-damaged group were more likely to choose high-denotative regions than scrambled regions as objects when differences could be observed on ipsilesional regions.
These results indicate that object recognition processes can operate on contralesional contours of ipsilesional regions even when object-centered attentional biases direct attention away from those contours. The finding that object recognition contributions to figure-ground segmentation behave like other cues to figure-ground that are thought to be computed preattentively lends support to the proposal that the object recognition processes relevant to figure-ground segmentation are also preattentive processes.

It is noteworthy that 1 participant in each of the braindamaged groups failed to show object recognition effects on their object decisions. Indeed, both of these participants saw scrambled ipsilesional regions as objects more often than high-denotative ipsilesional regions. Both participants showed the opposite effect for contralesional regions, where they saw high-denotative regions as objects more often than scrambled regions. Thus, overall, it seems as if these participants failed to show any effects on scene segmentation from object recognition processes (although there is not enough statistical power to put this statement to test). We consider the implications of the performance of these 2 participants in the Discussion section.

\section{Identification Accuracy}

Figure 4 shows conditional identification scores for each participant group and each side of the contour. The ANOVA revealed a main effect of group $[F(2,25)=6.29$,

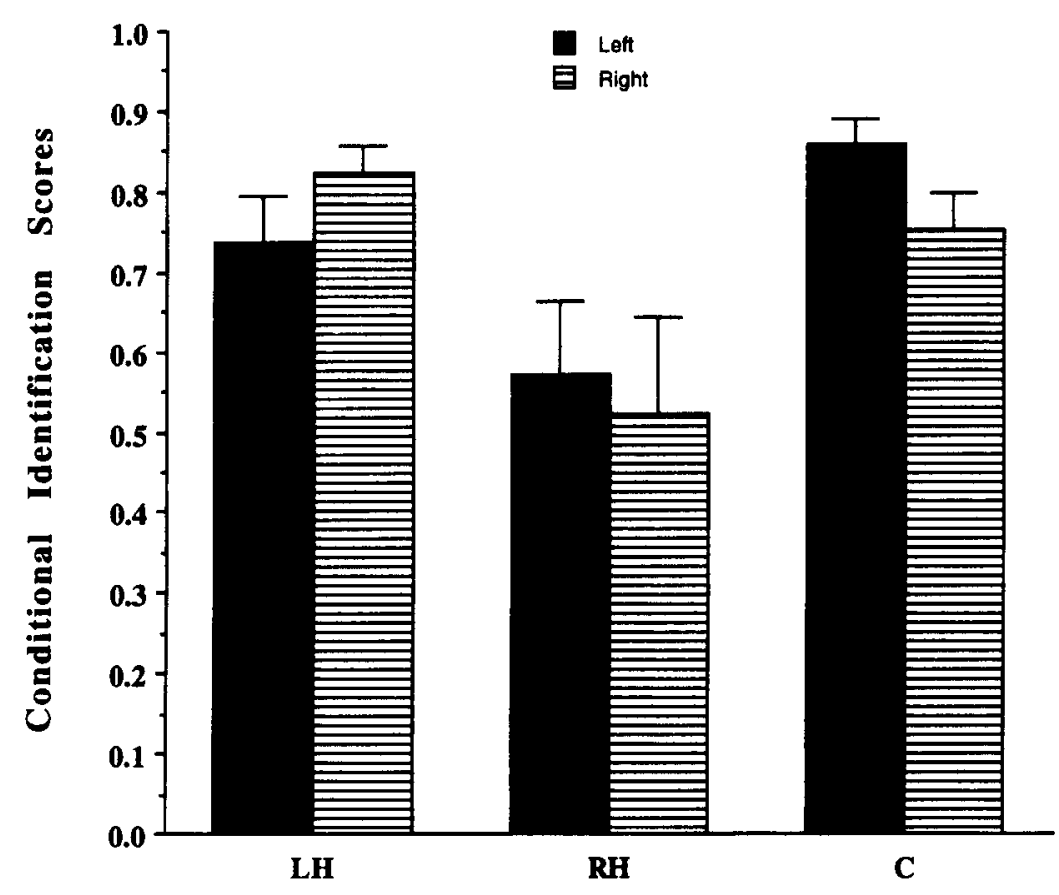

Figure 4. Conditional identification scores for left-hemisphere (LH)-damaged, right-hemisphere (RH)-damaged, and control participants. Black bars represent regions lying on the left of the central contour; striped bars indicate regions lying on the right of the central contour. 
$p<.005]$. No other main effects or interactions were significant ( $p$ s $>.12$ ). Tukey-Kramer post hoc tests showed that conditional identification scores were lower for the RH-damaged participants ( 0.55$)$ than for the LH-damaged participants $(0.78)$ or the control participants $(0.80)(p<$ $.05)$, but conditional accuracy scores for the $\mathrm{LH}$-damaged participants and the control participants did not differ statistically. Thus, the proportion of object decisions occurring without conscious identification of the object portrayed by the high-denotative region was larger for $\mathrm{RH}$-damaged than for LH-damaged and control participants. These results show that conscious object recognition does not necessarily accompany object recognition effects on scene segmentation. There were no statistically significant effects of contour side, although the patterns obtained are those that would be expected on an objectcentered attention account. These results suggest that the $\mathrm{RH}$ plays a role in the conscious identification of objects under the conditions employed here (i.e., conditions in which a good deal of amodal completion may be required). (For additional evidence that the RH may be specialized for amodal completion, see Corballis, Fendrich, Shapley, \& Gazzaniga, 1998.)

\section{DISCUSSION}

In the experiment reported here, object-centered attentional biases were clearly evident in both LH-damaged and RH-damaged participants who engaged in an object decision task involving figure-ground displays. Contrary to the predictions of a spatial attention account, brain-damaged participants were more likely to see contralesional regions than ipsilesional regions as objects in figure-ground displays. This pattern of performance indicates that attention is allocated within objects toward ipsilesional contours and away from contralesional contours. This object-centered attentional bias probably arises because the intact hemisphere is specialized for allocating attention contralaterally within an object (see ReuterLorenz et al., 1996).

One question investigated in the present experiment was whether object-centered attentional biases were more likely to be observed following damage to the $\mathrm{LH}$ or the RH. In previous investigations, in which the emphasis has been on separating object-centered biases from spatial attention biases, small numbers of participants with either RH or LH lesions have been tested, and different experimenters have used different tasks to probe for objectcentered attention (e.g., Behrmann \& Moscovitch, 1994; Driver et al., 1992; Humphreys \& Riddoch, 1994). In contrast, in this experiment, a relatively large number (16) of brain-damaged participants were tested on the same task, 8 with RH damage and 8 with LH damage. Objectcentered attentional biases were observed in both the LHand RH-damaged groups.

The two brain-damaged groups were similar on two indices of object-centered attentional bias (e.g., in both groups, a large overall proportion of object decisions favored contralesional regions as objects, and in both groups, the majority of participants were more likely to choose contralesional than ipsilesional high-denotative and scrambled regions as objects). However, the two braindamaged groups differed in that LH-damaged participants were more likely than $\mathrm{RH}$-damaged participants to see ipsilesional high-denotative and scrambled regions as objects. Taken as a whole, these results suggest that object-centered orienting biases may be stronger in the intact LH than in the intact RH, contrary to the proposal made by Humphreys and Riddoch (1994). This possibility requires further investigation. It is not clear, for example, whether or not the LH bias will be found to be stronger for all types of object-centered attention (e.g., objectcentered biases that are sensitive to the intrinsic handedness of an object (Behrmann \& Moscovitch, 1994; Behrmann \& Tipper, 1994; Tipper \& Behrmann, 1996).

A second question addressed in the present experiment was whether elderly control participants would show any attentional biases on the figure-ground task employed here. We found that the elderly control participants showed a slight, but statistically significant, bias to see left regions as objects. This slight attentional bias operated in the same direction as the object-centered attentional bias found in RH-damaged patients. Two possible interpretations can be offered for the bias observed in control participants. It might reflect a stronger object-centered attentional bias of the $\mathrm{LH}$ in the intact brain, or, alternatively, the bias might reflect aging-related damage to the brain. Although the former interpretation is consistent with other results obtained in this experiment (see above), the second interpretation cannot be ruled out, for a number of reasons. First, it is highly unlikely that evidence for hemispheric superiority would be obtained in normal participants under conditions involving long exposures and uncontrolled eye movements such as those used here. Therefore, the hemispheric differences evident in this experiment may not have derived from hemispheric superiority. Second, the hypothesis that the RH may decline faster than the LH as a function of age (Albert \& Moss; 1988; Goldstein \& Shelley, 1981) has recently received empirical support, at least for portions of the brain involved in some visual-perceptual tasks (e.g., Gerhardstein et al., 1998; Polster \& Rapcsak, 1994; Stark \& Coslett, 1993). Furthermore, Peterson and Gerhardstein (1998) found no bias toward seeing left regions as objects in young participants. The presence of a left bias in elderly participants that is absent in young participants is consistent with the differential aging hypothesis and not with the LH superiority hypothesis. We stress that the control data presented here do not support one hypothesis over the other. Further investigations of both elderly and young participants will be conducted to better explore object-centered attentional biases in the normal young and aging brains.

A third question addressed in the present paper was whether or not object recognition contributions to scene segmentation could be observed in brain-damaged patients who showed object-centered attentional biases. The answer was affirmative: Both groups of brain-damaged par- 
ticipants saw a larger proportion of high-denotative than scrambled ipsilesional regions as objects. Object recognition processes that affect the likelihood of seeing ipsilesional regions as objects operate on the contralesional contours of those regions, the same contours that are later ignored due to attentional biases.

These results constitute critical evidence in support of the proposal that a subset of object recognition processes operates preattentively and contributes to the assignment of object status. Of course, even preattentive processes might be ordered hierarchically, so the present results cannot rule out the possibility that within preattentive processing, the other scene segmentation cues are computed (fully or partially) before the critical object recognition processes are engaged. However, the present results, along with the results obtained from a visual agnosic patient (Peterson et al., 1998) can rule out the possibility that conscious object recognition is responsible for the evidence that high-denotative regions are more likely than scrambled regions to be seen as objects. Further empirical and modeling investigations are required to test the proposal that the early, preattentive, object recognition processes critical to scene segmentation do not require outputs from a scene segmentation stage before they can be engaged (Peterson, 1994a, 1994b, in press; Peterson \& Gibson, 1993, 1994a, 1994b).

One participant in each of the brain-damaged groups failed to show evidence of object recognition contributions to scene segmentation, whereas all of the other brain-damaged patients and all of the normal elderly control participants did. Peterson et al. (1998) reported a third such failure in a patient with bilateral lesions. All 3 patients who failed to show object recognition contributions to scene segmentation had lesions that involved the temporal lobe unilaterally. However, other patients with unilateral temporal lesions have shown normal object recognition contributions to scene segmentation (for examples, see Table 2 and Peterson et al., 1998). Further neuropsychological investigations and imaging experiments are necessary to identify the neural substrates for the preattentive object recognition processes under consideration here. The results of these investigations will be important for understanding both object recognition processes and scene segmentation processes.

More must be learned about the relationship between object-centered attention effects, such as those discussed in this article, and object-based attention effects. With respect to hemispheric specialization, Egly, Driver, and Rafal (1994) tested both RH- and LH-damaged patients for object-based attention effects, which are measured as a cost incurred for moving attention between, as opposed to within, objects (see, e.g., Baylis \& Driver, 1993; Duncan, 1984; Egly, Driver, \& Rafal, 1994; Egly, Rafal, et al., 1994). They obtained normal magnitude object-based effects in RH-damaged patients, regardless of whether the stimuli were presented in the LVF or the RVF. However, LH-damaged patients showed unusually large object-based attention effects for targets in the RVF and unusually small object-based attention effects for targets in the LVF. Egly an colleagues interpreted these results to indicate that the specialization for object-based attention is stronger in the LH than in the RH. Our results suggest that the LH specialization for object-centered attention may be stronger than that of the RH. Taken together, these findings call for an explication of the relationship between these two types of attention. One possibility is that the relative advantage for moving attention within, as opposed to between, objects (i.e., object-based attention) arises from object-centered attention. However, some investigators working on object-based attention consider the movement of attention within an object to be purely space based (Egly, Driver, \& Rafal, 1994; Egly, Rafal, et al., 1994). This poses a bit of a puzzle: Given that mechanisms devoted to object-centered attention exist, they might be expected to participate in moving attention within an object.

Egly, Rafal, et al. (1994) tested a split-brain patient and observed object-based attention effects for RVF (LH) presentation conditions, but not for LVF (RH). They took these results to indicate that object-based attention is uniquely mediated by the $\mathrm{LH}$, and not by the $\mathrm{RH}$. They argued that the object-based attention effects found in the LVF (RH) of both brain-damaged and normal control participants must be mediated by cross-callosal connections. It is not known whether cross-callosal connections are necessary for the object-centered attention effects observed in both the RH-damaged and LH-damaged participants in the experiment reported in this article. However, like the results obtained with normal participants (Reuter-Lorenz et al., 1996), the results of the present experiment suggest that both hemispheres mediate contralateral object-centered attentional biases.

\section{REFERENCES}

Albert, M. S., \& Moss, M. B. (1988). Geriatric neuropsychology. New York: Guilford.

Baylis, G. C., \& Driver, J. (1993). Visual attention and objects: Evidence for the hierarchical encoding of location hypothesis. Journal of Experimental Psychology: Human Perception \& Performance, 19. $451-470$.

BehrmanN, M., \& Moscovitch, M. (1994). Object-centered neglect in patients with unilateral neglect: Effects of left-right coordinates of objects. Journal of Cognitive Neuroscience, 6, 1-16.

BehrmanN, M., \& Tipper, S. P. (1994). Object-based visual attention: Evidence from unilateral neglect. In C. Umiltà \& M. Moscovitch (Eds.), Attention and performance XIV: Conscious and nonconscious processing and cognitive functioning (pp. 351-375). Cambridge, MA: MIT Press.

Caramazza, A., \& Hills, A. E. (1990). Spatial representation of words in the brain implied by studies of a unilateral neglect patient. Nature, 346, 267-269.

Corballis, P. M., Fendrich, R., Shapley, R., \& GazZaniga, M. (1998). $A$ dissociation between illusory contour perception and amodal boundary completion following callosotomy. Cognitive Neuroscience Society 1998 annual abstract program (p. 18). Cambridge, MA: MIT Press.

DERENZI, E. (1982). Disorders in space exploration and cognition. New York: Wiley.

Driver, J., Baylis, G. C., Goodrich, S. J., \& Rafal, R. D. (1994). Axisbased neglect of visual shapes. Neuropsychologia, 32, 1353-1365. 
Driver, J., Baylis, G. C., \& Rafal, R. D. (1992). Preserved figureground segregation and symmetry perception in visual neglect. $\mathrm{Na}$ ture, 360, 73-75.

Driver, J., \& HaLligan, P. W. (1991). Can visual neglect operate in object-centered coordinates? An affirmative single case study. Cognitive Neuropsychology, 8, 475-496.

DUNCAN, J. (1984). Selective attention and the organization of visual information. Journal of Experimental Psychology: General, 119, 501-517.

Egly, R., DRIVER, J., \& RAFAL, R. D. (1994). Shifting visual attention between objects and locations: Evidence from normal and parietal lesion subjects. Journal of Experimental Psychology: General, $\mathbf{1 2 3}$ 161-177.

Egly, R., Rafal, R., Driver, J., \& Starrveveld, Y. (1994). Covert orienting in the split brain reveals hemispheric specialization for object-based attention. Psychological Science, 5, 380-383.

Farah, M. J., BrunN, J. L., Wong, A. B., Wallace, M. A., \& CarPENTER, P. A. (1990). Frames of reference for allocating attention to space: Evidence from the neglect syndrome. Neuropsychologia, 28, 335-347.

Gainotti, G., Messerli, P., \& Tissot, R. (1972). Quantitative analysis of unilateral spatial neglect in relation to laterality of cerebral lesions. Journal of Neurology, Neurosurgery, \& Psychiatry, 38, 545-550.

Gerhardstein, P., Peterson, M. A., \& Rapcsak, S. Z. (1998). Agerelated hemispheric asymmetry in object discrimination. Journal of Clinical \& Experimental Neuropsychology, 20, 174-185.

Gibson, B. S., \& PeTERSON, M. A. (1994). Does orientation-independent object recognition precede orientation-dependent recognition? Evidence from a cueing paradigm. Journal of Experimental Psychology: Human Perception \& Performance, 20, 299-316.

Goldstein, G., \& Shelley, C. (1981). Does the right hemisphere age more rapidly than the left? Journal of Clinical Neuropsychology, 3 , 65-78.

HARROWER, M. R. (1936). Some factors determining figure-ground articulation. British Journal of Psychology, 26, 407-424.

HeILMAN, K. M., \& VAN DEN, A. (1980). Right hemisphere dominance for attention: The mechanism underlying hemispheric asymmetries of inattention (neglect). Neurology, 30, 327-330.

HochBeRG, J. (1971). Perception I: Color and shape. In J. W. Kling \& L. A. Riggs (Eds.), Woodworth and Schlossberg's Experimental psychology (3rd ed., pp. 395-474). New York: Holt, Rinehart \& Winston.

Hoffman, D. D., \& Richards, W. A. (1985). Parts of recognition. In S. Pinker (Ed.), Visual cognition (pp. 65-96). Cambridge, MA: MIT Press.

Humphreys, G. W., \& Riddoch, M. J. (1994). Attention to withinobject and between-objects spatial representations: Multiple sites for visual selection. Cognitive Neuropsychology, 11, 207-241.

Kahneman, D., Treisman, A., \& GibBs, B. J. (1992). The reviewing of object files: Object-specific integration of information. Cognitive Psychology, 24, 175-215.

KinsBouRne, M. (1970). The cerebral basis of lateral asymmetries in attention. Acta Psychologia, 33, 193-201.

KöHLER, W. (1947). Gestalt psychology. New York: New American Library. (Original work published 1929)

Marshall, J.C., \& Halligan, P. W. (1994). The yin and yang of visuospatial neglect: A case study. Neuropsychologia, 32, 1037-1057.

Mattingly, J. B., Price, M. C., \& Driver, J. (1996). Figure-ground segmentation and visual neglect. Cognitive Neuroscience Society 1996 annual meeting abstract program (p. 104). Cambridge, MA: MIT Press.

Mennemeier, M., Vezey, E., Chatterjee, A., Rapcsak, S. Z., \& Heilman, K. M. (1997). Contributions of left and right cerebral hemispheres to line bisection. Neuropsychologia, 35, 703-715.

Milner, A. D., Brechmann, M., \& Pasgliarini, A. (1992). To halve and halve not: An analysis of line bisection judgments in normal subjects. Neuropsychologia, 30, 515-526.

Neisser, U. (1967). Cognitive psychology. New York: Appleton Century Crofts.

OGDEN, J. A. (1987). The neglected left hemisphere and its contribution to visuo-spatial neglect. In M. Jeannerod (Ed.), Neurophysiological and neuropsychological aspects of spatial neglect (pp. 215-235). Amsterdam: North-Holland.

PALMER, S., \& ROCK, I. (1994). Rethinking perceptual organization:
The role of uniform connectedness. Psychonomic Bulletin \& Review, 1, 29-55.

Peterson, M. A. (1994a). The proper placement of uniform connectedness. Psychonomic Bulletin \& Review, 1, 509-514.

Peterson, M. A. (1994b). Shape recognition can and does occur before figure-ground organization. Current Directions in Psychological Science, 3, 105-111.

Peterson, M. A. (in press). What's in a stage name? Journal of Experimental Psychology: Human Perception \& Performance.

Peterson, M. A., de Gelder, B., Rapcsak, S. Z., Gerhardstein, P. C., \& BACHOUD-LÉvı, A. C. (1998). Intact object recognition effects on figure-ground segmentation in a visual agnosic. Investigation $O p h$ thalmology \& Visual Science, 39, 1870.

Peterson, M. A., \& Gerhardstein, P. C. (1998). Object-centered attentional biases in the left and right hemispheres in young and elderly normal observers. Manuscript in preparation.

Peterson, M. A., \& Gibson, B. S. (1993). Shape recognition contributions to figure-ground organization in three-dimensional display. Cognitive Psychology, 25, 383-429.

Peterson, M. A., \& Gibson, B. S. (1994a). Must shape recognition follow figure-ground organization? An assumption in peril. Psychological Science, 5, 253-259.

Peterson, M. A., \& Gibson, B. S. (1994b). Object recognition contributions to figure-ground organization: Operations on outlines and subjective contours. Perception \& Psychophysics, 56, 551-564.

Peterson, M. A., Harvey, E. H., \& Weidenbacher, H. L. (1991). Shape recognition inputs to figure-ground organization: Which route counts? Journal of Experimental Psychology: Human Perception \& Performance, 17, 1075-1089.

Peterson, M. A., Rapcsak, S. Z., \& Gerhardostein, P. (1994). Deficits in pre-figural recognition processes in patients with unilateral lesions: A new distinction. Cognitive Neuroscience Society 1994 annual meeting abstract program (p. 108). Cambridge, MA: MIT Press.

Polster, M. R., \& RAPCSAK, S. Z. (1994). Hierarchical stimuli and hemispheric specialization: Two case studies. Cortex, 30, 487-497.

Reuter-Lorenz, P. A., Drain, M., \& Hardy-Morais, C. (1996). Object-centered attentional biases in the intact brain. Journal of Cognitive Neuroscience, 8, 540-550.

Reuter-Lorenz, P. A., Kinsbourne, M., \& Moscovitch, M. (1990). Hemispheric control of spatial attention. Brain \& Cognition, 12, 240-266.

Rubin, E. (1958). Figure and ground. In D. Beardslee \& M. Wertheimer (Eds. and Trans.), Readings in perception (pp. 35-101). Princeton, NJ: Van Nostrand. (Original work published 1915)

SNOdgRass, J. G., \& VANDERWART, M. (1980). A standardized set of 260 pictures: Norms for name agreement, image agreement, familiarity, and visual complexity. Journal of Experimental Psychology: Human Learning \& Memory, 6, 174-215.

Stark, M. E., \& Coslett, H. B. (1993). The "Navon effect"-Forest before trees? It depends on how old you are and how many trees are in the forest [Abstract]. Journal of Clinical \& Experimental Neuropsychology, 15, 46.

TIPPER, S. P., \& BEHRMANN, M. (1996). Object-centered not scene-based visual neglect. Journal of Experimental Psychology: Human Perception \& Performance, 22, 1261-1278.

VeCERA, S. P., \& O'Reilly, R. C. (1998). Figure-ground organization and object recognition processes: An interactive account. Journal of Experimental Psychology: Human Perception \& Performance, 24, 441-462.

WALLACH, H. (1949). The role of memory in visual perception. Journal of Personality, 18, 6-13.

\section{NOTES}

1. The latter is a form of scene-based spatial neglect (Baylis \& Driver, 1993; Kahneman, Treisman, \& Gibbs, 1992).

2. A region can be a figure along one part of its boundary and a ground along another part of its boundary.

3. When they made this proposal, Humphreys and Riddoch (1994) acknowledged that contradictory evidence existed.

4. Egly and his coauthors (Egly, Driver, \& Rafal, 1994: Egly, Rafal, Driver, \& Starrveveld, 1994) observed an LH specialization for objectbased attention, which is not the same as object-centered attention. 
Object-based attention effects entail a cost incurred for switching attention between two objects as opposed to a single object. Although object-based attention effects are almost certainly related to object-centered attention effects, they are not identical. This paper is concerned with object-centered rather than object-based attention effects. Therefore, we will reserve further discussion of object-based attention effects for the Discussion.

5 . The high-denotative regions and the scrambled regions were matched in convexity and area as well-factors that are relevant to figure-ground segmentation (Harrower, 1936; Hochberg, 1971; Rubin, 1915/1958).

6. Other tests comparing upright to inverted orientations of the experimental stimuli also served as a critical foundation for these proposals (Peterson \& Gibson, 1994a, 1994b; Peterson et al., 1991).

7. It was proposed that the outputs from these object recognition processes also combine with monocular and binocular depth cues (Peterson, 1994a, 1994b; Peterson \& Gibson, 1993, 1994a).
8. No differences were observed as a function of set.

9. The data from 2 patients whose behavior differed as a function of spatial location are not presented in this paper. Both patients showed evidence of object-centered attentional biases, but these biases were modulated by spatial location. Since the purpose of this study was not to show that object-centered attentional biases can coexist with spatial attention biases, those 2 participants were eliminated.

10. We thank Jon Driver for pointing out in April 1995 that the effects we observed in the RH-damaged neglect patients we originally tested could be understood as an instance of object-centered attention.

(Manuscript received May 1, 1998; revision accepted for publication August 19, 1998.) 